\title{
RoboCup Humanoid Challenge: That's One Small Step for A Robot, One Giant Leap for Mankind
}

\author{
Hiroaki Kitano \\ Sony Computer Science Laboratory \\ 3-14-13 Higashi-Gotanda, Shinagawa \\ Tokyo 141 Japan \\ kitano@csl.sony.co.jp
}

\author{
Minoru Asada \\ Adaptive Machine Systems \\ Graduate School of Engineering \\ Osaka University, Yamadaoka, Suita \\ Osaka 565-0871 Japan \\ asada@ams.eng.osaka-u.ac.jp
}

\begin{abstract}
The ultimate goal of the RoboCup Initiative is to build a humanoid soccer team which beats a human World Cup Champion team. In this paper, we presents reasons why this goal should be pursued, and analyze technical issues involved in humanoid to play soccer game. The analysis demonstrates the breadth of technologies need to be developed through the course of the Challenge, which has major impacts to industries in general.
\end{abstract}

\section{Introduction}

RoboCup, The Robot World Cup Initiative, is an international research initiative to foster robotics and AI technologies using soccer / football as a common task [10]. Already over 1,000 researchers through the world is participating in various aspects of the RoboCup Initiative. Currently, games are played by wheel-based robots, soccer game by humanoid robot is the next major leap in the field which leads to the ultimate goal of the RoboCup. We proposed that the ultimate goal of the RoboCup Initiative to be stated as follows:

By mid-21st century, a team of fully autonomous humanoid robot soccer players shall win the soccer game, comply with the official rule of the FIFA, against the winner of the most recent World Cup.

We propose that this goal to be the one of the grand challenges shared by robotics and AI community for next 50 years. This goal may sounds overly ambitious given the state of the art technology today. Nevertheless, we believe it is important that such a long range goal to be claimed and pursued. It took only 50 years from the Write Brother's first aircraft to Apollo mission to send man to the moon and safely return them to the earth. Also, it took only 50 years, from the invention of digital computer to the Deep Blue, which beat human world champion in chess. We recognize, however, that building humanoid soccer player requires equally long period and extensive efforts of broad range of researchers, and the goal will not be met in any near term.

The successful landmark project claims to accomplish a very attractive and broadly appealing goals. The most successful example is the Apollo space program. In case of the Apollo project, the U.S. committed the goal of "landing a man on the moon and returning him safely to earth." [9] The accomplishment of the goal itself marks the history of the mankind. Although the direct economic impact of having someone landed on the moon is $\operatorname{slim}^{1}$, technologies developed to achieve this goal was so significant that it formed the powerful technological and human foundations to the American industries. The important issue for the landmark project is to set the goal high enough so that a series of technical breakthrough is necessary to accomplish the task, and the goal need to be widely appealing and exciting. In addition, a set of technologies necessary to accomplish the goal must be the technologies which can form the foundation of the next generation industries. In the Apollo project, the actual goal was much more than manned mission to the

\footnotetext{
${ }^{1}$ To be fair, the Apollo mission was planned to gain the "National Prestige" and to demonstrate technical superiority over the former Soviet Union. Even in this, aspect, no direct military advantage was gained by having few astronauts on the moon.
} 
moon ${ }^{2}$.

In this paper, we argue that RoboCup Humanoid Challenge is a significant long range goal of the intelligent robotics community, and has major industrial impacts as its spill over effects.

\section{Justification}

Obviously, in order to claim real robot soccer, it have to be done by humanoid robot, which can run fast, kick and dribble a ball, and jump to try miracle heading shot. Humanoid players has to be biped robot, just like actual human players. However, the real question that ought to be asked is "what is a potential impacts of developing humanoid soccer players?" Two major issues need to be justified to warrant RoboCup Humanoid Challenge;

- Why do we want to build humanoid?

- Why do we use a soccer game as a driving force?

\subsection{Why Humanoid?}

Humanoid research is certainly a dream of robotics researchers, and many robotics laboratories has been working on the project [14]. The success of the Honda Humanoid P2 and P3 [6] have changed the standard and the perspectives that the community should pursue. Biped walking humanoid robot is now possible, and we expect numbers of universities and corporate research laboratories will soon manage to build their own biped humanoid robots. Within a decade the humanoid will be the norm, and the issue will be the quality of their behaviors, such as how fast it can move, how flexible it can move and turn, can it jump, and others.

We believe that social and industrial impacts of humanoid robot is far reaching and deserves major research investments. One of the major advantages in humanoid robot as opposed to wheel-based robot is

\footnotetext{
2PROJECT APOLLO: "THAT'S ONE SMALL STEP FOR A MAN, ONE GIANT LEAP FOR MANKIND." The national effort that enabled Astronaut Neil Armstrong to speak those words as he stepped onto the lunar surface, fulfilled a dream as old as humanity. But Project Apollo's goals went beyond landing Americans on the Moon and returning them safely to Earth: To establish the technology to meet other national interests in space; To achieve preeminence in space for the United States; To carry out a program of scientific exploration of the Moon; and To develop man's capability to work in the lunar environment. (http://www.ksc.nasa.gov/history/apollo/apollo-goals.txt)
}

\begin{tabular}{|l|l|l|}
\hline & Chess & RoboCup \\
\hline \hline Environment & Static & Dynamic \\
\hline State Change & Turn taking & Real time \\
\hline Info. accessibility & Complete & Incomplete \\
\hline Sensor Readings & Symbolic & Non-symbolic \\
\hline Control & Central & Distributed \\
\hline
\end{tabular}

Table 1: Comparison of Chess and RoboCup

its flexible mobility. While the mobility of the wheelbased robot is limited to flat surface or slopes, humanoid robots can step onto uneven terrane and climb up and down stairways. Humanoid robots can maneuver into sphere of our daily life where wheel-based robot cannot enter [6]. The research will be focused on humanoid robot which can work with human with sharing the same office space, public spaces, and in personal houses.

The use of multiple leg robots may augment the terrain that robot can enter. However, these robots only move themselves by negotiating with rough terrains, and no capacity for a manipulation task while navigation such as running catch of flying objects. If such a robot is designed to do such tasks, additional hand-like manipulator on top of a multiple-leg robot would be necessary and therefore cost ineffective.

Early experiences in designing wheel-based robot$\mathrm{s}$ for RoboCup revealed that wheel-based robots are inherently limited in sophisticated ball handling, unless they attach kicking and dribbling devices which mimick human legs.

\subsection{Why Soccer?}

¿From the perspectives of artificial intelligence research, computer chess has been one of the most symbolic target of challenge. The question on whether computers can be "intelligent" enough to beat human world champion was clearly answered by the accomplishment of the Deep Blue team in 1997. AI community continue to challenge for new landmark challenge target, and it is now in a good agreement that soccer is suitable for the next long range target. Behind such an agreement is that difference of characteristics between Chess and soccer games (Table 1). Domain characteristics in the RoboCup Soccer domain demonstrates sharp contrasts to computer chess, and considered to generate founder mental technologies for the next wave of industries.

Robotics community, on the other hand, has always struggled with real physics, vision, and other problems. Nevertheless, the task of multiple mobile robots 
that cooperatively carry out missions under adversarial environment has not extensively investigated in the past, except for military purposes. However, when robots are used in daily life, it must be able to learn and understand how to collaborate with other robots and human. The element of multiple agent cooperation is one of the critical factor in RoboCup.

In addition, soccer is a good task for developing humanoid with high level of mobility and flexible behaviors. Obviously, humanoid players must be able to walk, run, jump, and kick a ball very quickly. Also, it must be able to cope with severe contact from other robots or human. Traditionally humanoid robot research assumes that there is no contact between the robot and other robot or human. Even if it is assumed, the contact is extremely limited and assumed to be non-hostile. This assumption no longer hold$\mathrm{s}$ once we decide to deploy robots in real world. In the office, all sort of things happen. People may accidentally bump into the robot at the corner when the robot is carrying fragile materials. A robot should be able to quickly avoid collision, or to balance its body so that the fragile object it is carrying would not be damaged. It would be much safer for both robot and human. RoboCup offers ideal test-bed because soccer game inevitably involves contacts.

Educational effects is yet another major factors in using soccer as a target task. Through the RoboCup initiative, we already learned that students are extremely excited, involved, and motivated when RoboCup was used for course material, project task, and thesis projects. In addition, there are few cases that students apply to certain university which has RoboCup team, because they wish to participate in RoboCup. This is because RoboCup captures imagination of these students. We consider this is very important aspect, since long-term success of the robotics and AI field depends on the involvement of talented and dedicated new members of the community.

\section{$3 \quad$ Research Issues}

\subsection{Materials and Basic Components}

\subsubsection{Surface Materials}

Robot must have soft surface material, so that it will not hurt human or other objects. However, at the same time, there must be a mechanism which protects internal mechanics and electronics from external force. An artificial muscle with strength and speed equal to that of a human muscle may soon be possible although many fundamental physical and engineering questions remain [2]

\subsubsection{Frame Materials}

Apart from the surface material, a light weight, but strong frame material would be necessary to sustain itself and to protect robot's internal system from the external force. This could be organized like born of human body, or subsurface layered under the soft surface material.

\subsubsection{Power Supply}

Power supply systems, such as battery or other novel systems, which can sustain the activity of humanoid robot for at least 45 minutes is required. Currently, humanoid robots or other complex legged robot can operate only for 10-15 minutes with battery.

\subsubsection{Energy Saving Architecture}

Long operation time cannot be achieved only by the innovation of the supply-side. Drastically efficient low-power consumption systems, as well as effective energy-saving control mechanism need to be developed. Any new development in this aspect of the technology is far reaching and undoubtfully benefits whole earth by mitigating the global environment and resource problems.

\subsubsection{Actuation Systems}

Current motor and gear system is to inefficient and fragile. Drastically new driving devices and joint mechanisms need to be developed. Although there are few artificial muscle projects, none of them reach the level of practical use. However, innovations in this area can spill over to entire industry.

\subsubsection{Mechanical Design}

Building humanoid to meet the challenge requires broad range of innovation in mechanical design. For example, one might wish to develop a new joint system which mimick human joint system. Also, it may be driven antagonistic artificial muscle groups. Highly robust, inexpensive, and flexible joint systems are one of challenges. 


\subsection{Basic Control Issues}

\subsubsection{High Performance Mobility}

Current humanoid systems have reached a point that walking at slow speed is possible. However, playing soccer game requires drastically higher mobility performance. How can we build a humanoid which can, for example, walk slowly, then speed up to run very fast, jump toward the ball, and stop. Jumping motion$\mathrm{s}$ was attained by a series of robots (not humanoid) that Marc Rai bert developed at MIT [11]. However, these robots and current humanoid robots operates on very different principles. There is a serious need to develop an integrated theory of mobility for biped robots.

\subsubsection{Behavioral Robustness}

Robots which dynamically interacts in the real world is always posed a danger of being fell down, twisted, or even get damaged by unexpected accidents. However, the robot shall be robust enough to recover the posture quickly to the normal operational mode. Whenever a part of body is damaged, it should adjust the remaining body movement to best accomplish the task. While it is not feasible to pre-program recipe for all possible cases, it is essential that built-in learning and adaptation scheme cope with the situation.

\subsubsection{Behavioral Complexity}

Combined motions is an essential elements in flexible and highly maneuvrable robotics systems. When people learn to play a new sports, we generate and learn a new set of combined motion sequences. This is essentially a learning of motor control. However, it shall be combined with sensory inputs and high level cognition.

\subsubsection{Human Control of High DOF Systems}

Humanoid robot is a high DOF system. Honda P-3, for example, has 30 DOF, and Waseda University's WABIAN has 43 DOF. When we wish to use such a platform by a tele-operation mode, controlling high DOF is important issue. Without any intelligent software layers, the operator must somehow control over $30 \mathrm{DOF}$ in real time. This is a major challenge in human computer interaction (HCI) in the new area.

\subsection{Sensory Systems}

\subsubsection{Vision}

The visual information is the richest source of information to perceive not only the external world but the effects of the robot actions as well. The Computer Vision researchers have been seeking for the accurate 3-D geometry reconstructed from 2-D visual information believing in that the 3-D geometry is the most powerful and general representation to be used in many applications such as view generation for video database and robot manipulation and navigation. However, the time-consuming 3-D reconstruction might not be necessary nor optimally encoded for the task given to the RoboCup player. In order to react to the situation in real time, the RoboCup player needs the information which behavior to select against which situation. This does not mean to build up a special-purpose vision system but to claim that vision is a part of complex system that interacts in specific ways with world The RoboCup is one of such worlds which make clear the role of vision and evaluate the performance of the image processing that have been left ambiguous in the computer vision field.

\subsubsection{Auditory System}

It is critically important for humanoid robot players to recognize auditory inputs. the auditory capability can be divided into two categories: (1) Speech Understanding, and (2) Auditory Scene Understanding [12]. In speech understanding, the humanoid shall understand spoken sentences of coaches or other human so that they can react based on what they heard. Auditory scene analysis is extremely useful technology. It means to understand that a certain sound is a sound that an oppo nent players is running behind you, or the sound of ball being kicked. The sound provides non-verbal information of the dynamical world.

\subsubsection{Other Sensing Systems}

Sensory system replicating force sensor of human skin would be essential. Currently, many robotics systems detects collision of its body parts

with other objects through motor systems such as using applied current and shift encoder readings. However, this can only detect aggregated torque applied to the body parts, and cannot detect subtle difference of materials or soft touch. 


\subsubsection{Sensor Fusion}

In addition to vision, the RoboCup player might need other sensing such as sonar, touch, and force/torque to discriminate the situations that cannot be discriminated from only the visual information nor covered by the visual information. Again, the RoboCup player need$\mathrm{s}$ the real time processing for multi-sensor fusion and integration. Therefore, the deliberative approaches to obtain the robust estimation by multi-sensor system does not seem suitable. We should develop a method of sensor fusion/integration for the RoboCup.

\subsubsection{Sensory-Motor Integration}

One of the essential process in formation of cognitive map, or concept formation, is association of sensory inputs and motor command. The global changes in visual field can be better handled when coupled with leg motions so that the changes can be attributed to motion of the robot itself. There is broad range of undiscovered research topic in this field. by having humanoid which can serve as a platform of perception and learning research, the scientific contribution can be enormous.

\subsection{High Level Cognitive Systems}

\subsubsection{Strategy Planning}

Strategy planning also exists in wheel-based robot players. However, the humanoid players will have much larger strategic options due to increased ball handing and maneuvaling capability.

In the wheel-based robot, at least in the early stage of RoboCup. most robots simply hit the ball at the frontal part of the robot. Humanoid, however, can dribble the ball, kick the ball to the side or back, spin the ball to create a curved trajectory. In addition, it can receive the ball before the goal and throw the ball into the field by using his/her hands.

Due to drastically increased tactical and behavioral options, strategic planning must cope with magnitude larger search space, and larger number of branching factors at any moment.

\subsubsection{Learning}

Learning of behaviors and tactic would be the major issue. There are numbers of specific areas learning is essential, such as basic low-level motion control and coordination sequence generation, association of preceptory inputs and motion control, learning of coordination of high DOF system, and strategy acquisition.
It is eesnetial that such learning research to be carried out on a robotics platform which has rich perception inputs and high DOF. Current learning and robotics perception research is limited due to simple preceptory input and motion modalities.

\subsubsection{Brain and Cognitive Science}

Much of issues in high-level cognition for humanoid robot players may be shared with that of wheel-based robots. Such issues are general architecture for strategy planning and re-planning, real-time reasoning, and other abstract strategy and tactics. In humanoid players, however, the possible strategic option may increase drastically associating with the degree of freedom the body has. This issue is coupled with the question "does intelligence requires body?" Brooks argued that embedment is critical to intelligence [4], however, the DOF of the robot he developed was so small that the claim was not clearly supported. The Cog project [3] has high DOF with upper body of humanoid. In reality, the degree of freedom the body has and the level of intelligence is closely related. Single cellular organism can hardly seen as having intelligence. $E$. Coli. Even in multi-cellular organism, plants do not have degree of freedom it can actively control. Some researchers already pointed out the important of using physical body with high DOF [1]. Creating humanoid and other robots with high degree of freedom and mobility is an ideal test-bed of cognitive science and artificial intelligence research. The question can be translated as: "Through the course of evolution, is there any need for living system without high degree of freedom to develop any level of intelligence?"

\section{The Load to World Cup}

\subsection{Humanoid League}

Unlike NASA, RoboCup itself do not have research funding. RoboCup is like academic conferences just like IROS and IJCAI, and administered by The RoboCup Federation, which is a non-profit scientific and cultural copration in Bern Switzerland. In order to encourage the challenge and promote research and education, RoboCup provides forum of technical exchange, exhibitions, and compeititons. Before, actually play soccer with human players, RoboCup organize humanoid leagues in following categories:

Fully Autonomous Humanoid League: Soccer games by teams of fully autonomous humanoid 
biped robots. A regular league will be performed by humanoid robots of height equivalent to real human. Should proposal be made to create other size of humanoid league, such as smaller size, such a league may be created.

Tele-operation Humanoid League: Soccer games by teams of tele-operated humanoid robots. The operator is allowed to control the robot only through the information obtained by sensors onboard the robot.

Virtual Humanoid League: Soccer game by teams of simulated humanoid robots, with high quality computer graphics, accurate physics simulation, and vision and sensor simulation. Such a finegrain simulation is shown to be extremely useful in understanding cognitive aspects of animals [13]. Hodgins and Wooten [7] have developd an animation system of human athletes in which male and female models performs dynamic athletic behaviors such as running, bicycling, and vaulting

\subsection{Benchmark}

However, organizing actual competition by humanoid is not possible in any near term. Instead, we propose to organize a series of benchmark of basic behaviors to evaluate the progress of mobility performance. The benchmark shall include basic behaviors, such as walk, run, change speed, turn, and jump, as well as cognitive performance such as object recognition, and object tracking. In addition, combined behaviors such as object following, collision avoidance, kick a stationary ball, kick a moving ball, and receive a moving ball shall be defined. Concurrently, challenges on cognitive issues such as auditory systems, planning and learning shall be coordinated and integrated in future. Overall pathways of the benchmark set is shown in Figure 1.

\subsection{Standard Components and Software}

One of the problems which hamper rapid progress of robotics is absence of standard components and software compatibilities. Most robot components are home-made and components are hardly exchangeable with other systems. Defining standard which allows exchange of design and components would greatly enhance the progress in the field. Currently, OPENR [5] is proposed to be the standard for high DOF mobile and intelligent robots. Also, in a priority research program on Intelligent Robotics by Japanese Ministry of
Education, several common equipments such as fourleg robot base and DSP vision system [8] have been developed and a nnumber of research groups have been using them and exchaging information useful for their robotics research.

\section{Conclusion}

This paper proposed RoboCup Humanoid Challenge, and analyzed technical issues, possible scientific contributions, as well as justifications on why we should promote this challenge. We believe that the challenge proposed has a long term and high impact goal, which could generate major spill-over effects. Industrial, scientific and educational impacts would be enormous.

After all, one might ask "Can we actually accomplish the goal?" Despite broad range of problems that have to be solved, we believe that the goal can be accomplished, and the humanoid will far surpasses motion capability of human being someday. As in the case of chess, machines can out perform specific capabilities of human being ${ }^{3}$. Also, robots will not have stamina loss, or mental unstability ${ }^{4}$. When humanoid surpasses human capability in soccer domain, we will have highly robust and reliable machinery that would support human activities in the way which is unthinkable today.

\section{References}

[1] Asada, M., "An Agent and an Environment: A View on "Having Bodies" - A Case Study on Behavior Learning for Vision-Based Mobile Robot," Proceedings of 1996 IROS Workshop on Towards Real Autonomy, pp.19-24, 1996.

[2] Brock, D.L., "Review of Artificial Muscle Based on Contractile Polymers," MIT AI Memo No. 1330, November 1991.

\footnotetext{
${ }^{3}$ Garry Kasparov himself noted "I can out-calculate any player in the world, but I cannot out-calculate the machine".

${ }^{4}$ Frederic Friedel, Garry Kasparov's technical advisor, said "The problem is that the machine can play in a play that should make it easy for Garry to win, but it is still forcing him to difficult calculations. That takes a lot of energy, and I don't know if Garry can keep that up for six games." He also mentioned psychological advantage of machine. When people lost the game, the human player often suffer destructive impacts, however, Friedel commented that "but Deep Blue doesn't know that it lost."
} 


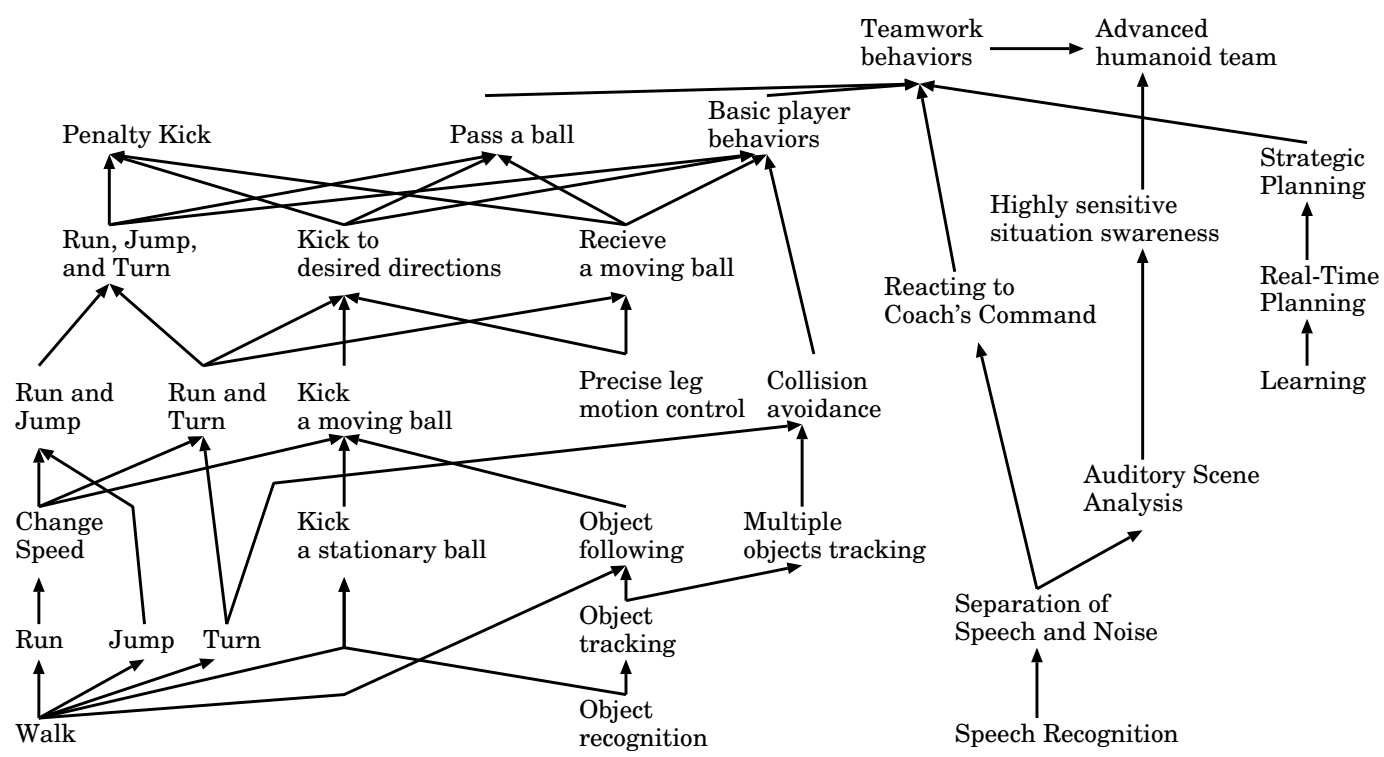

Figure 1: Benchmark Flow

[3] Brooks, R. and Stein, L. A., "Building Brains for Bodies," A.I. Memo no. 1439, MIT, Cambridge, 1993.

[4] R. Brooks, "A robust layered control system for a mobile robot," IEEE J. Robotics and Automation, RA-2, 14-23, April, 1986.

[5] Fujita, M. and Kageyama, K., "An Open Architecture for Entertainment Robot," In Proceedings of the First International Conference on Autonomous Agents, 1997.

[6] Hirai, K., "Current and Future Perspectivce of Honda Humanoid Robot," Proc. of IROS-97, Grenoble, 1997.

[7] Hodgins, J. K., and Wooten, W. L., "Animating Human Athletes," Preprint of 8Th ISRR, pp.147154, 1997.

[8] Inoue, H., "Research Program on Intelligent Robotics: A Priority Research by Japanese Ministry of Education," Preprint of 8Th ISRR, pp.40-41, 1997.

[9] Kennedy, J. F., "Urgent National Needs," Speech to a Joint Session of Congress, 25 May 1961, Congressional Record - House (25 May 1961) p.8276.

[10] Kitano, H., Asada, M., Kuniyoshi, Y., Noda, I., Osawa, E., and Matsubara, H., "RoboCup:
A Challenge AI Problem," AI Magazine, Spring 1997.

[11] Raibert, M., Legged Robots That Balance, The MIT Press, 1986.

[12] Rosenthal, D. and Okuno, H.G., (Eds.) Computational Auditory Scence Analysis, Lawrence Erlbaum Associates, 1997.

[13] Tu, X., and Terzopoulos, D. "Artificial Fishes: Physics, Locomotion, Perception, Behavior," Proc. of SIG GRAPH-94, Orland, Florida, July 24-29, pp43-49, 1994.

[14] Yamaguchi, J. and Takanashi, A., "Design of Biped Walking Robots Having Antagonistic Driven Joints Using Nonlinear Spring Mechanism," Proc. of IROS-97, Grenoble, 1997. 